\title{
BMJ Open Early Chronic Kidney Disease Care Programme delays kidney function deterioration in patients with stage I- IIIa chronic kidney disease: an observational cohort study in Taiwan
}

\author{
Shu-Fen Niu, ${ }^{1,2,3}$ Chung-Kuan Wu (D) , ${ }^{4,5}$ Nai-Chen Chuang, ${ }^{6}$ Ya-Bei Yang, ${ }^{7}$ \\ Tzu-Hao Chang 8
}

To cite: Niu S-F, Wu C-K, Chuang N-C, et al. Early Chronic Kidney Disease Care Programme delays kidney function deterioration in patients with stage I-Illa chronic kidney disease: an observational cohort study in Taiwan. BMJ Open 2021;11:e041210. doi:10.1136/ bmjopen-2020-041210

- Prepublication history and supplemental material for this paper is available online. To view these files, please visit the journal online (http://dx.doi org/10.1136/bmjopen-2020041210).

C-KW and T-HC contributed equally.

Received 06 June 2020 Revised 30 December 2020 Accepted 07 January 2021
Check for updates

(C) Author(s) (or their employer(s)) 2021. Re-use permitted under CC BY-NC. No commercial re-use. See rights and permissions. Published by BMJ.

For numbered affiliations see end of article.

Correspondence to Dr Chung-Kuan Wu; m008533@ms.skh.org.tw and Professor Tzu-Hao Chang; kevinchang@tmu.edu.tw

\section{ABSTRACT}

Objectives To investigate the effect of the Early Chronic Kidney Disease (CKD) Care Programme on CKD progression in patients with CKD stage I-Illa.

Design Observational cohort study.

Setting Taipei Medical University Research Database from three affiliated hospitals.

Participants Adult non-pregnant patients with CKD stage I-Illa from Taipei Medical University Research Database between 1 January 2012 and 31 August 2017 were recruited. These patients were divided into Early CKD Care Programme participants (case) and non-participants (control). The models were matched by age, sex, estimated glomerular filtration rate and CKD stage with 1:2 propensity score to reduce bias between two groups. Outcome measures The risks of CKD stage I-IIla progression to IIIb between Early CKD Care Programme participants and non-participants.

Results Compared with the control group, the case group demonstrated more comorbidities and higher proportions of hypertension, diabetes mellitus, gout, dyslipidaemia, heart disease and cerebrovascular disease, but had lower risk of progression to CKD stage Illb before and (HR 0.72; $95 \% \mathrm{Cl} 0.61$ to 0.85 ) and after (adjusted HR (aHR) 0.67; $95 \% \mathrm{Cl} 0.55$ to 0.81 ) adjustments. Moreover, Kaplan-Meier analysis revealed the cumulative incidence of CKD stage IIIb was significantly lower in the case group than in the control group. Finally, the programme was an independent protective factor against progression to stage Illb, especially in patients with CKD stage Illa before (HR 0.72; $95 \% \mathrm{Cl} 0.61$ to 0.85 ) and after (aHR $0.67 ; 95 \% \mathrm{Cl} 0.55$ to 0.81 ) adjustments.

Conclusions The Early CKD Care Programme is an independent protective factor against progression of early CKD.

\section{INTRODUCTION}

Chronic kidney disease (CKD) is recognised as a global health concern ${ }^{1}$ because its overall prevalence in the general population is $>10 \%$. CKD prevalence ranged from $11.7 \%$ to $15.1 \%$, with stages $\mathrm{I}-\mathrm{V}$ accounting for

\section{Strengths and limitations of this study}

- The study provides the information on the preventive effect of the Early CKD Care Programme on CKD progression.

- The patients in our study were recruited from the greater Taipei area, which might not be representative of all clinical situations in Taiwan because of the urban-rural medical disparity.

- Selection bias should be considered for participants owing to their motivation and role of medical personnel.

- The clinical outcome focuses on the progression of early CKD, rather than major adverse cardiac events or mortality.

$2.8 \%-4.2 \%, 2.7 \%-5.3 \%, 6.4 \%-8.9 \%, 0.3 \%-$ $0.5 \%$ and $0.1 \%$, respectively. Therefore, most patients with CKD are in the early stages, ${ }^{2}$ in which they may have no symptoms or signs. ${ }^{3}$ Such early disease stages are not easily discovered and diagnosed by primary care physicians; therefore, most patients had not consulted a nephrologist, with fewer than $6 \%$ of the patients had consulting a nephrologist even for stage III CKD. ${ }^{4}$ Without appropriate response or management of early CKD, it progresses to advanced CKD and finally to end-stage renal disease (ESRD), which usually requires management with dialysis. Therefore, exponential growth in medical $\operatorname{costs}^{5}$ is expected. Despite treatment, patients with ESRD have poor quality of life and high mortality risk. ${ }^{6}$

In CKD stages I-II, an optimal outcome can be achieved with adequate assessment, diagnosis and treatment. ${ }^{4}$ The US Centers for Disease Control and Prevention recommends that early CKD progression prevention should include testing for and controlling CKD risk 
factors as well as maintaining a healthy weight through a balanced diet and physical exercise. ${ }^{7}$ Moreover, early monitoring and treatment in conjunction with lifestyle adjustments can improve the revisit rate of patients with CKD and delay renal function reduction. ${ }^{8}$

In Taiwan, $>85000$ patients require dialysis and the related National Health Insurance (NHI) expenditure reached NT $\$ 44.9$ billion in 2017 . To reduce kidney function deterioration, improve the quality of life, reduce the burden on the NHI programme and achieve the goal of prioritising prevention over management, Taiwan's Ministry of Health and Welfare launched the Early CKD Care Programme aimed at active management of stage I-IIIa CKD. ${ }^{9}{ }^{10}$ However, the effectiveness of intervention in delaying kidney function deterioration warrants exploration. Therefore, this study explored the effects of an intervention-based Early CKD Care Programme in reducing kidney function deterioration in patients with stage I-IIIa CKD.

\section{MATERIALS AND METHODS \\ Data source}

This cohort study obtained information on patients with CKD stages I-IIIa in the institutional and clinical research database of Taipei Medical University (CRDB). This database contains the electronic health and medical records of $>3$ million patients from three affiliated hospitals, namely Taipei Medical University Hospital, Wan Fang Hospital and Shuang Ho Hospital.

\section{Study design and cohort}

Figure 1 illustrates the patient selection process for the study cohort. From the CRDB, we identified patients with CKD who had more than two medical return visits between 1 January 2012 and 31 August 2017. We enrolled those who met the following criteria of CKD stages I-IIIa: patients with normal renal function but who present signs of kidney damage such as proteinuria, haematuria and other conditions with estimated glomerular filtration rate $(\mathrm{eGFR}) \geq 90 \mathrm{~mL} / \mathrm{min} / 1.73 \mathrm{~m}^{2}$; patients with kidney damage with eGFR $60-89.9 \mathrm{~mL} / \mathrm{min} / 1.73 \mathrm{~m}^{2}$ and patients with eGFR $45-59.9 \mathrm{~mL} / \mathrm{min} / 1.73 \mathrm{~m}^{2}$, respectively. We excluded patients aged $<18$ years and those who were pregnant. The remaining patients were divided into the case group, those who participated in the Early CKD Care Programme with P4301C, P4302C or P403603C treatment codes, and the control group, consisting of those who did not participate in the Early CKD Care Programme.

\section{Outcome measures and comorbidity}

Major comorbidities diagnosed before the index date, according to claims data, were defined as baseline comorbidities. The comorbidities were identified using the International Classification of Diseases, Ninth Revision, Clinical Modification codes for hypertension, diabetes mellitus (DM), gout, hyperlipidaemia, heart disease and cerebrovascular disease, as shown in online supplemental

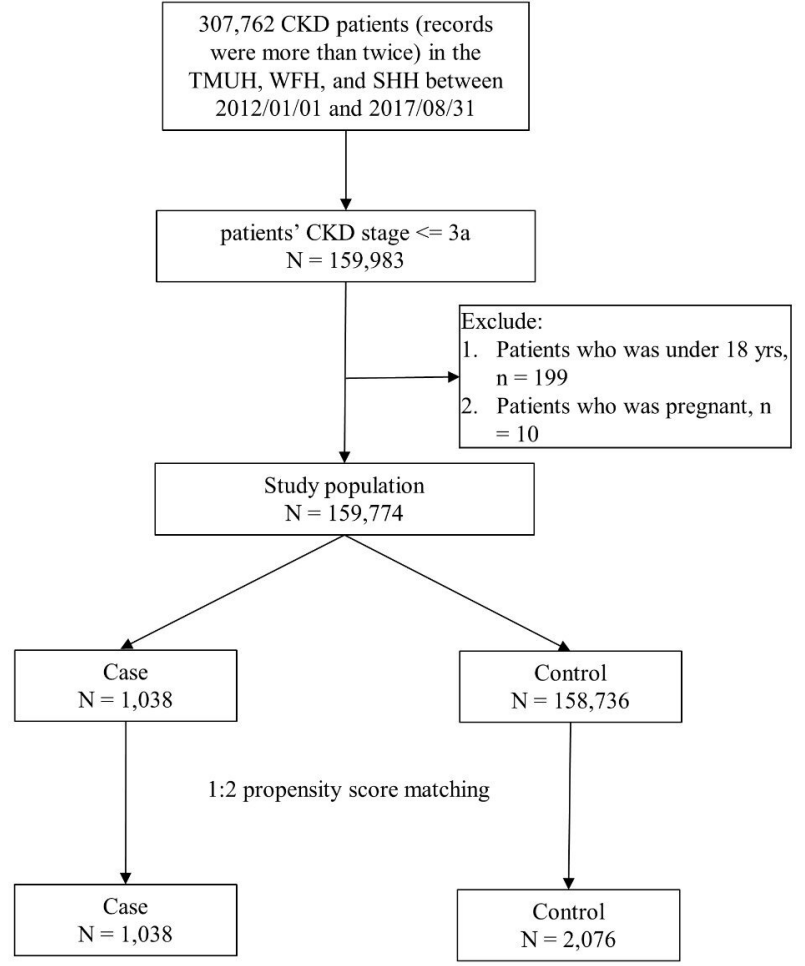

Figure 1 Flow of patient selection for the study cohort. From January 2012 to August 2017, 307762 patients with chronic kidney disease (CKD) with more than two visits to the participating hospitals were identified in Taipei Medical University Research Database. Adult non-pregnant patients with CKD who met the specific criteria of stage I-IIla CKD were regarded as patients with early CKD. Those who participated in the Early CKD Care Programme comprised the case group, and those not participating in the programme served as the control group. We conducted 1:2 propensity score matching with age, sex, estimated glomerular filtration rate and CKD stage to reduce selection bias in the control group. SHH, Shuang Ho Hospital; TMUH, Taipei Medical University Hospital; WFH, Wan Fang Hospital.

table S1. Other baseline demographic data included age, sex, eGFR, CKD stage and number of comorbidities. Here, the eGFR was calculated as $186 \times$ creatinine-1.154×age-0.203 ( $\times 0.742$ for female), and the number of comorbidities was defined as the sum of the aforementioned comorbidities in the year prior to the enrolment date. The outcome of the study was patient progression to CKD stage IIIb during the study period.

\section{The Early CKD Care Programme}

The Bureau of NHI in Taiwan launched the Early CKD Care Programme in 2011. Patients who participated in the programme constitute this study's case group. The programme involved (i) referral to a nephrologist and provision of medication for hypertension, diabetes and hyperlipidaemia to delay kidney function deterioration, avoid damage caused by improper medication and prevent complications; (ii) CKD case managers enrolled these patients and provided nursing education and lifestyle consultations and routinely monitored disease 
progress and conducted renal function tests, urinalysis and urine albumin-creatinine or protein-creatinine ratio evaluations. The CKD case managers informed the doctors and patients' families regarding medical practice and care-giving. The nursing education provided during the enrolment period included the following: (i) teaching the basic structure and functions of kidneys; (ii) introducing the common symptoms of kidney conditions as well as the examination values; (iii) explaining daily care and prevention of kidney conditions; (iv) communicating the importance of routine monitoring; (v) communicating the importance of consulting a doctor before using medication; (vi) introducing kidney needle biopsy; (vii) introducing hyperlipidaemia, hypertension, DM, kidney conditions and their complications and (viii) explaining dietary instructions. Lifestyle recommendations included the following: smoking cessation; weight loss, particularly for those with BMI $>25 \mathrm{~kg} / \mathrm{m}^{2}$ or men and women with a waist circumference of $>90$ and $>80 \mathrm{~cm}$, respectively; daily protein intake $<1.5 \mathrm{gm} / \mathrm{kg}$; prevention of routine or excessive alcohol consumption; adequate exercise and daily salt intake $<100 \mathrm{mEq}$. Routine physical examinations were conducted at least once every 6 months for CKD stages I-IIIa and urine protein, urine creatinine, serum creatinine, low-density lipoprotein and haemoglobin Alc were tested. The control group received routine care and was not enrolled or monitored by CKD case managers.

\section{Statistical analysis}

Descriptive statistics were used to summarise the demographic data. Continuous variables are presented as mean and $\mathrm{SD}$, and categorical variables are presented as the number of enrollees and percentage (\%). The models were matched by age, sex, eGFR and CKD stage with 1:2 propensity score to reduce bias between the case group and the control group. Considering that the number of participants in the case group $(n=1038)$ were substantially smaller than those in the control group $(n=158$ 736), we chose a greedy and nearest neighbour matching for propensity score matching (PSM) algorithm. Before PSM, we used Student's t-test to assess age and eGFR; and the $\chi^{2}$ test or Fisher's exact test were used for sex, CKD stage, number of comorbidities, hypertension, DM, gout, heart disease, hyperlipidaemia and cerebrovascular disease. After PSM, we evaluated the differences between matched pairs using the signed rank test for continuous data and McNemar's test for binary data. Multivariable Cox proportional hazards models were matched to all the candidate variables, including comorbidity numbers, hypertension, DM, gout, hyperlipidaemia, heart disease and cerebrovascular disease to determine the risk factors for patients progressing to CKD stage IIIb. Subgroup analysis was used to determine the risk factors for patients progressing to CKD stage IIIb from baseline CKD stage IIIa or the stages before it. A two-sided statistical test at $5 \%$ significance was used. Analyses were performed using SAS (V.7.11; SAS Institute, Cary, North Carolina, USA).

\section{Patient and public involvement}

The study used de-identified data from the institutional and Taipei Medical University Research Database. No patients were involved in developing the research question or in determining the outcome measures. Patients were not involved in designing the study. There are no plans to disseminate the results of this study to any participants.

\section{RESULTS}

\section{Study population characteristics}

Table 1 presents the characteristics of the study population. Before PSM, a total of 159774 patients with stage I-IIIa CKD were enrolled from the participating hospitals, including 1038 in the case group and 158736 in the control group. All the variables were significantly different between the two groups (all $\mathrm{p}<0.001$ ). Age was significantly higher in the case group than in the control group. By contrast, eGFR was significantly lower in the case group than that in the control group. The proportions of sex, CKD stage IIIa, hypertension, DM, gout, hyperlipidaemia, heart disease, cerebrovascular disease and proportion of number of comorbidity were significantly higher in the case group than in the control group. To reduce bias, 1:2 PSM was used to match the age, sex, eGFR and CKD stage. After PSM, 3114 patients with stage I-IIIa CKD from the participating hospitals during the study period were finally enrolled in the study, including 1038 in the case group and 2076 in the control group. The proportion of hypertension, DM, gout, heart disease, hyperlipidaemia, cerebrovascular disease and proportion of number of comorbidities remained significantly higher in the case group than in the control group (all $\mathrm{p}<0.001$ ). Distribution of eGFR among cases and controls during the follow-up period is shown in online supplemental table S2.

\section{Association of Early CKD Care Programme and risk factors with early CKD progression}

Table 2 lists the crude HRs and adjusted HRs (aHRs) of all variables for stage I-IIIa CKD that progressed to CKD stage IIIb during the study period. Compared with patients in the control group, the HR for progression to CKD stage IIIb was 0.72 (95\% CI 0.61 to 0.85 ) for those participating in the Early CKD Care Programme. After adjustments for the variables listed in table 1, those in the control group still exhibited significant risk for progression to CKD stage IIIb (aHR 0.67; 95\% CI 0.55 to 0.51 ). In addition, DM, heart disease or cerebrovascular disease in patients with stage I-IIIa CKD were significant risk factors for progression to CKD stage IIIb. The KaplanMeier curves for the cumulative incidence of progression to CKD stage IIIb was significantly higher in patients with stage I-IIIa CKD who did not participate in the Early CKD Care Programme (control group) than the curves in those who participated in the programme (case group) during the follow-up period (log-rank test, $\mathrm{p}=0.0025$; figure 2 ). 
Table 1 Baseline characteristics of enrollees

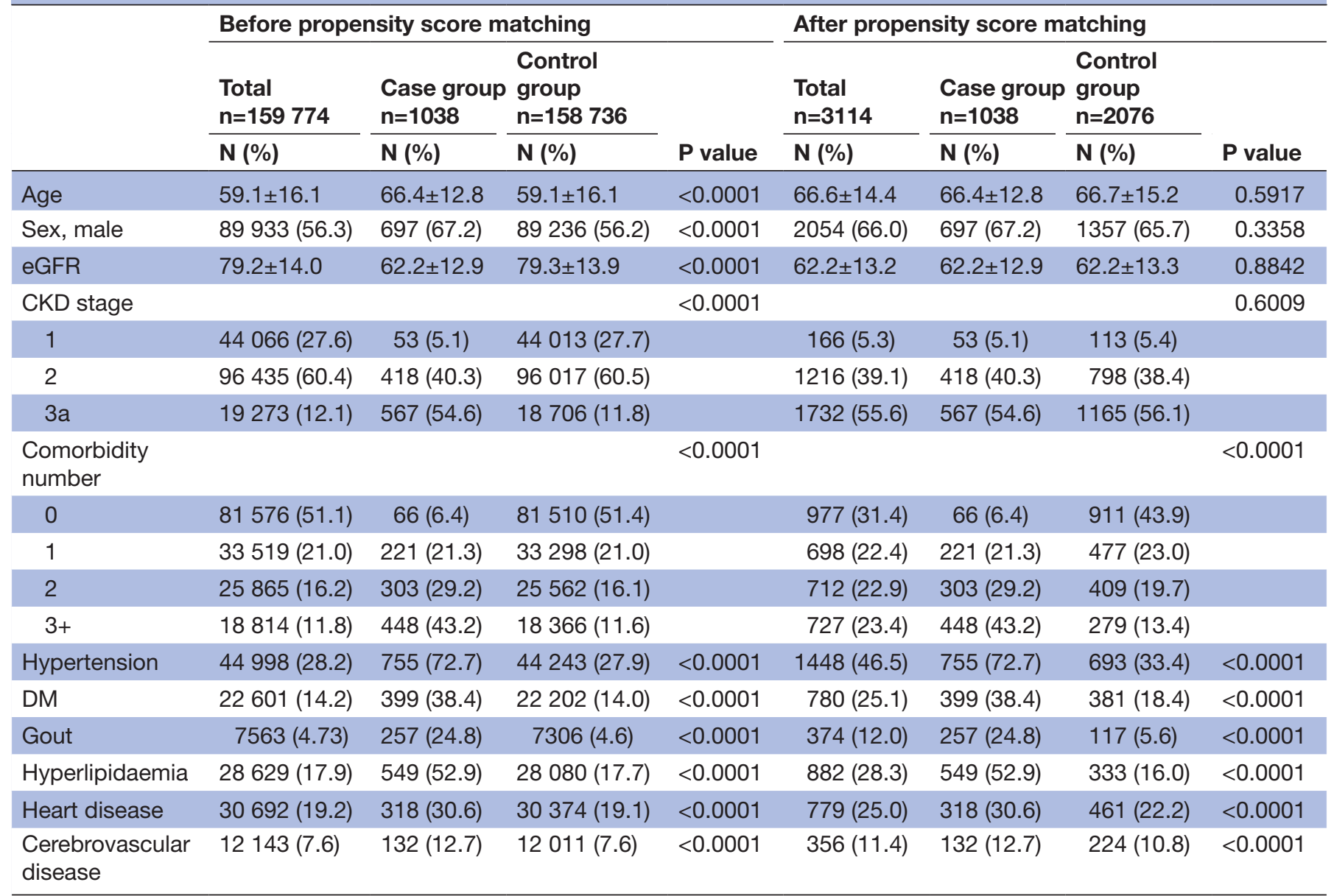

Matched variables were age, sex, eGFR and CKD stage.

CKD, chronic kidney disease; DM, diabetes mellitus; eGFR, estimated glomerular filtration rate.

The median follow-up duration was 3.0 (95\% CI 1.0 to 4.7) years. Deterioration to CKD stage IIIb within 1, 3 and 5 years was respectively noted 374,563 and 644 patients in the control group and 140, 217 and 234 patients in the case group.

\section{Association of Early CKD Care Programme and risk factors between CKD stage I-II and CKD stage IIla with early CKD progression}

Of the 3114 patients with stage I-IIIa CKD in this study, 1382 patients with CKD stages I-II and the remaining 1732 patients were in stage IIIa. Table 3 lists the crude HRs and aHRs of all variables for the progression of CKD from stage I-IIIa to IIIb during the study period. In the CKD stages I-II subgroup, the Early CKD Care Programme, the number of comorbidities and comorbid hypertension, DM, gout, heart disease, hyperlipidaemia and cerebrovascular disease had no significant influence on the progression of CKD from stage I-II to IIIb even after adjustment for the variables. However, in the stage IIIa CKD subgroup, compared with those in the control group, the HR for progression to CKD stage IIIb in those with participated in the Early CKD Care Programme was 0.72 (95\% CI 0.60 to 0.87$)$. After adjustments for the variables listed in table 1, participation in the programme remained a significant protective factor against progression to CKD stage IIIb (aHR 0.67; 95\% CI 0.55 to 0.81 ). In addition, compared with patients with stage IIIa CKD but without DM, those with DM were at a greater risk of progression to CKD stage IIIb (HR 1.26; 95\% CI 1.01 to 1.57 and aHR 1.69; $95 \%$ CI 1.16 to 2.47 ). Compared with patients without heart disease with CKD stage IIIa, those with heart disease with CKD stage IIIa were at a greater risk for progression to CKD stage IIIb after adjustment for the variables (aHR 1.65; 95\% CI 1.12 to 2.45 ).

\section{DISCUSSION}

In this clinical observational study, we demonstrated that patients with stage I-IIIa CKD who participated in the Early CKD Care Programme exhibited significantly delayed deterioration of renal function to CKD stage IIIb compared with non-participants, particularly those patients in stage IIIa. We also observed that DM, heart disease and cerebrovascular disease are risk factors for deterioration of renal function in patients with stage IIIIa CKD. 
Table 2 Univariable and multivariable Cox regression analysis for the risk of CKD I-IIla progression to CKD IIIb among the Early CKD Care Programme and other risk factors $(n=3114)$

\begin{tabular}{|c|c|c|c|c|}
\hline & \multicolumn{2}{|c|}{ Univariable } & \multicolumn{2}{|c|}{ Multivariable* } \\
\hline & $\begin{array}{l}\text { HR } \\
(95 \% \mathrm{Cl})\end{array}$ & $P$ value & $\begin{array}{l}\text { aHR } \\
(95 \% \mathrm{Cl})\end{array}$ & $P$ value \\
\hline \multicolumn{5}{|l|}{ Group } \\
\hline Control & ref & & ref & \\
\hline Case & $\begin{array}{l}0.72(0.61 \\
\text { to } 0.85)\end{array}$ & $<0.0001$ & $\begin{array}{l}0.67(0.55 \\
\text { to } 0.81)\end{array}$ & $<0.0001$ \\
\hline
\end{tabular}

Comorbidity number

\begin{tabular}{|c|c|c|c|c|}
\hline 0 & ref & & ref & \\
\hline 1 & $\begin{array}{l}0.99(0.77 \\
\text { to } 1.29)\end{array}$ & 0.9687 & $\begin{array}{l}0.80(0.54 \\
\text { to } 1.17)\end{array}$ & 0.2438 \\
\hline 2 & $\begin{array}{l}0.94(0.73 \\
\text { to } 1.21)\end{array}$ & 0.6374 & $\begin{array}{l}0.54(0.29 \\
\text { to } 1.02)\end{array}$ & 0.0592 \\
\hline $3+$ & $\begin{array}{l}1.16(0.93 \\
\text { to } 1.46)\end{array}$ & 0.1931 & $\begin{array}{l}0.46(0.17 \\
\text { to } 1.23)\end{array}$ & 0.1195 \\
\hline
\end{tabular}

\begin{tabular}{cllll}
\multicolumn{4}{c}{ Hypertension } \\
No & ref & & ref & \\
Yes & $\begin{array}{l}0.98(0.82 \\
\text { to } 1.16)\end{array}$ & 0.7803 & $\begin{array}{l}1.24(0.85 \\
\text { to } 1.81)\end{array}$ & 0.2654 \\
\hline DM & & & & \\
No & ref & & ref & \\
Yes & $1.30(1.07$ & 0.0075 & $1.72(1.23$ & 0.0015 \\
& to 1.58$)$ & & to 2.41$)$ & \\
Gout & & & & \\
No & ref & & ref & \\
Yes & $0.86(0.67$ & 0.2577 & $1.25(0.87$ & 0.2241 \\
& to 1.11$)$ & & to 1.77$)$ & \\
\hline
\end{tabular}

Hyperlipidaemia

$\begin{array}{lllll}\text { No } & \text { ref } & & \text { ref } & \\ \text { Yes } & 0.98(0.81 & 0.8467 & \begin{array}{l}1.26(0.88 \\ \text { to } 1.80)\end{array} & 0.2152 \\ & \text { to } 1.19) & & \end{array}$

\begin{tabular}{|c|c|c|c|c|}
\hline \multicolumn{5}{|c|}{ Heart disease } \\
\hline No & ref & & ref & \\
\hline Yes & $\begin{array}{l}1.29(1.06 \\
\text { to } 1.58)\end{array}$ & 0.0132 & $\begin{array}{l}1.70(1.20 \\
\text { to } 2.40)\end{array}$ & 0.0027 \\
\hline
\end{tabular}

Cerebrovascular disease

\begin{tabular}{lllll} 
No & ref & \multicolumn{3}{c}{ ref } \\
Yes & $1.36(1.04$ & 0.027 & $1.59(1.12$ & 0.0104 \\
& to 1.78) & & to 2.27$)$ & \\
\hline
\end{tabular}

${ }^{*}$ The multivariable model was adjusted for all variables. aHR, adjusted HR; CKD, chronic kidney disease; DM, diabetes mellitus; ref, reference.

Compared with the control group, the case group had a higher mean age, a lower eGFR, a higher proportion of CKD stage IIIa and more comorbidities before PSM. In the real-life clinical scenario, these disparities are reasonable. First, patients with stage I and II CKD typically have

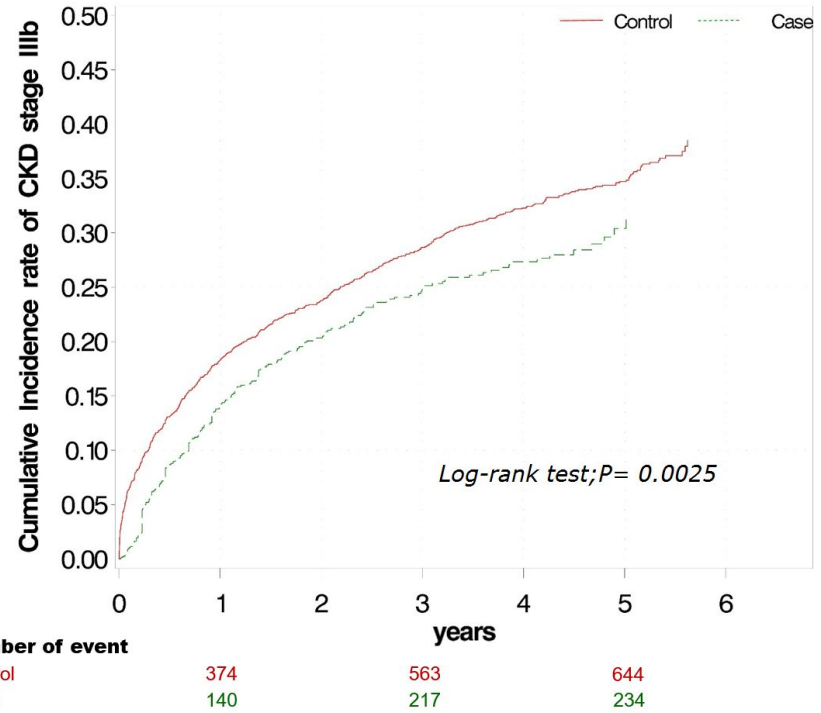

Figure 2 Cumulative incidence of progression to chronic kidney disease (CKD) stage IIIb in patients with stage I-IIla CKD in case and control groups. Kaplan-Meier analysis revealed that the cumulative incidence of progression to CKD stage IIlb was significantly higher in patients with stage I-IIla CKD who did not participate in the Early CKD Care Programme compared with that of those who participated in the programme, during the follow-up period (log-rank test, $p=0.02$ ).

no noticeable symptom ${ }^{4}$; hence, they are usually not referred to a nephrologist. Second, patients with CKD stage IIIa are more likely to manifest clinical symptoms than patients with earlier stages of the disease and, therefore, consult a nephrologist or seek medical attention. Third, patients with CKD IIIa with more comorbidities are more likely to be referred to a nephrologist than are those with fewer comorbidities. Fourth, older patients with more comorbidities are also more likely to be referred to specialists than younger patients with same comorbidities. CKD managers frequently encourage patients with clinical symptoms and those who consulted a nephrologist, have more comorbidities or are older to participate in the Early CKD Care Programme. Therefore, PSM was used to match variables such as age, sex, eGFR and CKD stage to reduce the bias of basic characteristics between the two groups during further analysis.

After PSM, we observed that the case group still showed more comorbidities such as hypertension, DM, gout, dyslipidaemia, heart disease and cerebrovascular disease, than the control group. Hypertension and CKD are closely interlinked. Uncontrolled hypertension can accelerate CKD progression ${ }^{11}$; thus, blood pressure control is essential to prevent CKD progression. ${ }^{12} \mathrm{DM}$ is also a major cause of CKD and a risk factor for CKD progression. ${ }^{13-15}$ Compared with those without DM, patients with DM have a 3.8-fold higher risk of developing CKD. ${ }^{15}$ Among patients with type $2 \mathrm{DM}, 42.3 \%$ have kidney injury. ${ }^{2}$ Compared with CKD patients without DM, those with DM developed CKD earlier and experienced more severe CKD complications. ${ }^{16}$ Intracellular hyperglycaemic leads 
Table 3 Univariable and multivariable Cox regression analysis for the risk of baseline stage $<3$ a progression to stage $3 \mathrm{~b}$ and stage 3a progression to stage $3 \mathrm{~b}$ among the Early Chronic Kidney Disease Care Programme and other risk factors

\begin{tabular}{|c|c|c|c|c|c|c|c|c|}
\hline & \multicolumn{4}{|c|}{ Baseline stage $<3 a n=1382$} & \multicolumn{4}{|c|}{ Baseline stage $=3 a n=1732$} \\
\hline & \multicolumn{2}{|c|}{ Univariable } & \multicolumn{2}{|l|}{ Multivariable* } & \multicolumn{2}{|c|}{ Univariable } & \multicolumn{2}{|l|}{ Multivariable* } \\
\hline & HR $(95 \%$ Cl) & $P$ value & aHR $(95 \% \mathrm{Cl})$ & $P$ value & HR (95\% Cl) & $P$ value & aHR $(95 \% \mathrm{Cl})$ & $P$ value \\
\hline \multicolumn{9}{|l|}{ Group } \\
\hline Control & ref & & ref & & ref & & ref & \\
\hline Case & 0.75 (0.52 to 1.08$)$ & 0.1244 & 0.75 (0.48 to 1.17$)$ & 0.2059 & 0.72 (0.60 to 0.87$)$ & 0.0005 & $0.34(0.51$ to 0.80$)$ & $<0.0001$ \\
\hline \multicolumn{9}{|c|}{ Comorbidity number } \\
\hline 0 & ref & & ref & & ref & & ref & \\
\hline 1 & 0.61 (0.33 to 1.12$)$ & 0.109 & 0.50 (0.20 to 1.26$)$ & 0.142 & 1.09 (0.82 to 1.46$)$ & 0.5593 & $0.87(0.56$ to 1.35$)$ & 0.5345 \\
\hline 2 & 0.82 (0.49 to 1.38$)$ & 0.4528 & 0.59 (0.14 to 2.48$)$ & 0.4753 & 0.95 (0.71 to 1.27$)$ & 0.7322 & $0.51(0.25$ to 1.05$)$ & 0.0664 \\
\hline $3+$ & 0.99 (0.57 to 1.72$)$ & 0.9617 & 0.64 (0.06 to 6.54$)$ & 0.7025 & 1.18 (0.91 to 1.52$)$ & 0.2062 & $0.39(0.13$ to 1.20$)$ & 0.1013 \\
\hline \multicolumn{9}{|c|}{ Hypertension } \\
\hline No & ref & & ref & & ref & & ref & \\
\hline Yes & 0.93 (0.63 to 1.36$)$ & 0.6964 & $1.10(0.45$ to 2.66$)$ & 0.7025 & $0.99(0.81$ to 1.20$)$ & 0.8805 & 1.32 (0.86 to 2.03$)$ & 0.2019 \\
\hline \multicolumn{9}{|l|}{ DM } \\
\hline No & ref & & ref & & ref & & ref & \\
\hline Yes & 1.48 (0.94 to 2.34$)$ & 0.0932 & 1.99 (0.87 to 4.54$)$ & 0.1032 & 1.26 (1.01 to 1.57$)$ & 0.0385 & 1.69 (1.16 to 2.47$)$ & 0.0065 \\
\hline \multicolumn{9}{|l|}{ Gout } \\
\hline No & ref & & ref & & ref & & ref & \\
\hline Yes & $0.79(0.43$ to 1.46$)$ & 0.4536 & 1.05 (0.45 to 2.43$)$ & 0.9181 & 0.89 (0.67 to 1.19$)$ & 0.4329 & $1.34(0.90$ to 1.99$)$ & 0.1525 \\
\hline \multicolumn{9}{|c|}{ Hyperlipidaemia } \\
\hline No & ref & & ref & & ref & & ref & \\
\hline Yes & $0.73(0.48$ to 1.11$)$ & 0.1404 & 0.76 (0.34 to 1.70$)$ & 0.5014 & 1.07 (0.87 to 1.33$)$ & 0.5204 & 1.52 (0.99 to 2.30$)$ & 0.0507 \\
\hline \multicolumn{9}{|c|}{ Heart disease } \\
\hline No & ref & & ref & & ref & & ref & \\
\hline Yes & $1.20(0.74$ to 1.93$)$ & 0.4599 & 1.47 (0.70 to 3.12$)$ & 0.3093 & 1.24 (0.99 to 1.56$)$ & 0.0618 & 1.65 (1.12 to 2.45$)$ & 0.0124 \\
\hline \multicolumn{9}{|c|}{ Cerebrovascular disease } \\
\hline No & ref & & ref & & ref & & ref & \\
\hline Yes & 1.78 (0.97 to 3.28$)$ & 0.0644 & 1.89 (0.84 to 4.26$)$ & 0.1247 & 1.25 (0.92 to 1.70$)$ & 0.1602 & $1.48(0.99$ to 2.21$)$ & 0.0576 \\
\hline
\end{tabular}

*The multivariable model was adjusted for all variables.

aHR, adjusted HR; DM, diabetes mellitus.

to endothelial dysfunction, increased oxidative stress and protein accumulation on the vascular wall, which cause vascular complications. ${ }^{17}$

In addition to hypertension and DM, gout is independently associated with CKD. ${ }^{18}$ Patients with hyperuricaemia are particularly susceptible to gout development. Hyperuricaemia is an independent risk factor for CKD, and hyperuricaemia treatment may delay CKD progression. ${ }^{19} 20$ Chronic hyperuricaemia is associated with hypertension, metabolic syndrome, CKD and cardiovascular disease. ${ }^{21}$ Dyslipidaemia is a risk factor for CKD, and CKD causes alterations in the lipoprotein profile. Therefore, the dyslipidaemia-CKD relationship is reciprocal. ${ }^{22}$ Hypertension, DM and dyslipidaemia are major causes of cardiovascular and cerebrovascular disease in patients with CKD. Treatment of hypertension, DM and dyslipidaemia in patients with CKD can reduce the occurrence of cardiovascular events and improve associated outcomes. ${ }^{23}$ Given the links between these diseases, the high proportion of heart and cerebrovascular disease observed in the case group may be expected. In theory, CKD in patients with many comorbidities should progress more rapidly from stage I-IIIa to IIIb than those with fewer comorbidities. However, in our study, despite having more comorbidities, the case group had better renal outcomes than the control group. Therefore, the Early CKD Care Programme may be assumed to be instrumental in delaying renal function deterioration.

The effect of the Early CKD Care Programme on the progression of CKD from early stages to stage IIIb was analysed. We found that participation in the programme significantly delayed the progression of CKD from stage IIIa to IIIb, however, we also observed no significant results for the progression of CKD from stages I-II to IIIb. 
Although the case group had low HRs for stage IIIb CKD compared with the control group, this difference was nonsignificant. CKD progression from stage I-II to IIIb may require some time, which could explain why few patients in the control group with stage I-II CKD progressed to stage IIIb during the follow-up period. Although some studies have developed clinical predication models for $\mathrm{CKD}$, the study groups in these investigations generally had stage III-IV CKD and ESRD was defined as the outcome. ${ }^{24} 25$ No clinical predication model has yet been designed for stages I-II or IIIa-IIIb CKD. Further investigation employing clinical predication models for earlyto-advanced CKD are warranted. Figure 2 illustrates that the protective effect of the Early CKD Care Programme was sustained over the follow-up period, although the difference in cumulative incidence rate between the two groups gradually increased. The decrease of the slope over time may be attributed to the fact that patients who overcame the decline of their eGFR to $<45$ for over 1 year had good compliance or few comorbidities.

In our clinical study, patients with stage I-IIIa CKD with DM, heart disease or cerebrovascular disease exhibited considerable risk of progression to stage IIIb CKD. These results are similar to the findings of the Kidney Early Evaluation Programme ${ }^{4}$ and a population-level cohort study by Tonelli et $a l .{ }^{26}$ Besides other conditions, DM and heart disease are also significant risk factors for the progression of CKD from stage IIIa to IIIb. Therefore, in addition to the Early CKD Care Programme, the Diabetes Shared Care Programme, which has been proven to reduce cardiovascular and cerebrovascular events and mortality risks, ${ }^{27}$ may be implemented.

The current study had some limitations that may affect the interpretation of the results. First, the CRDB only included data from three educational medical institutions located in New Taipei City and Taipei City in Taiwan. The greater Taipei area has adequate medical resources and, thus, may not be representative of all clinical situations in Taiwan on account of the urban-rural medical disparity. Second, our study cannot completely eliminate concerns related to selection bias because this phenomenon may be attributed to multiple reasons, including differential rates of death, and cause-specific models could feature assumptions that do not necessarily resolve competing risk issues. Third, the clinical outcome of our study was limited to the progression of early CKD; this work does not provide a comprehensive assessment of cardiovascular events and mortality. Fourth, the study did not take the potential effects of reversible kidney injury into account. Finally, the ethnicity of most of Taiwan's population is Chinese; thus, the results may not be generalisable to populations of other ethnic backgrounds.

In conclusion, the results of this study revealed that patients with stage I-IIIa CKD who participated in the Early CKD Care Programme benefit from a reduction in renal function deterioration. As such, this programme should be promoted and implemented, especially among those with stage IIIa CKD. More research is needed to understand what type of participants in the Early CKD Care Programme and which aspects of the programme yield the more effective results.

\section{Author affiliations}

${ }^{1}$ Department of Nursing, Shin Kong Wu Ho-Su Memorial Hospital, Taipei, Taiwan

${ }^{2}$ Department of Nursing, Fu Jen Catholic University, New Taipei, Taiwan

${ }^{3}$ College of Nursing, Taipei Medical University, Taipei, Taiwan

${ }^{4}$ Division of Nephrology, Department of Internal Medicine, Shin Kong Wu Ho-Su Memorial Hospital, Taipei, Taiwan

${ }^{5}$ School of Medicine, Fu Jen Catholic University, New Taipei, Taiwan

${ }^{6}$ Clinical Data Center, Office of Data Science, Taipei Medical University, Taipei,

Taiwan

${ }^{7}$ Division of Cardiovascular Surgery, Shin Kong Wu Ho-Su Memorial Hospital, Taipei, Taiwan

${ }^{8}$ Clinical Big Data Research Center, Taipei Medical University Hospital, Taipei, Taiwan ${ }^{9}$ Graduate Institute of Biomedical Informatics, College of Medical Science and Technology, Taipei Medical University, Taipei, Taiwan

Contributors All authors reviewed the manuscript. S-FN collected and interpreted the data and wrote the manuscript. N-CC and T-HC ran the data and performed statistical analyses. S-FN and C-KW determined the concept and design of this study. Y-BY contributed to the manuscript revision. T-HC and C-KW helped to write the manuscript and conceived the study.

Funding This study was supported by grant from the Ministry of Science and Technology, Taiwan (MOST 109-2314-B-341-003-MY3).

Competing interests None declared.

Patient consent for publication Not required.

Ethics approval The study was approved by the Institutional Review Board of Taipei Medical University (TMU-JIRB-201803022).

Provenance and peer review Not commissioned; externally peer reviewed.

Data availability statement Data are available from the institutional and clinical research database of Taipei Medical University (CRDB). Due to legal restrictions imposed by the government of Taiwan in relation to the 'Personal Information Protection Act', data cannot be made publicly available.

Supplemental material This content has been supplied by the author(s). It has not been vetted by BMJ Publishing Group Limited (BMJ) and may not have been peer-reviewed. Any opinions or recommendations discussed are solely those of the author(s) and are not endorsed by BMJ. BMJ disclaims all liability and responsibility arising from any reliance placed on the content. Where the content includes any translated material, BMJ does not warrant the accuracy and reliability of the translations (including but not limited to local regulations, clinical guidelines, terminology, drug names and drug dosages), and is not responsible for any error and/or omissions arising from translation and adaptation or otherwise.

Open access This is an open access article distributed in accordance with the Creative Commons Attribution Non Commercial (CC BY-NC 4.0) license, which permits others to distribute, remix, adapt, build upon this work non-commercially, and license their derivative works on different terms, provided the original work is properly cited, appropriate credit is given, any changes made indicated, and the use is non-commercial. See: http://creativecommons.org/licenses/by-nc/4.0/.

\section{ORCID iD}

Chung-Kuan Wu http://orcid.org/0000-0003-4446-0167

\section{REFERENCES}

1 Bello AK, Levin A, Tonelli M, et al. Assessment of global kidney health care status. JAMA 2017;317:1864-81.

2 Hill NR, Fatoba ST, Oke JL, et al. Global prevalence of chronic kidney disease - a systematic review and meta-analysis. PLoS One 2016;11:e0158765.

3 Stenvinkel P. Chronic kidney disease: a public health priority and harbinger of premature cardiovascular disease. J Intern Med 2010;268:456-67.

4 Agrawal V, Jaar BG, Frisby XY, et al. Access to health care among adults evaluated for CKD: findings from the kidney early evaluation program (KEEP). Am J Kidney Dis 2012;59:S5-15. 
5 Mills KT, Xu Y, Zhang W, et al. A systematic analysis of worldwide population-based data on the global burden of chronic kidney disease in 2010. Kidney Int 2015;88:950-7.

6 Webster AC, Nagler EV, Morton RL, et al. Chronic kidney disease. Lancet 2017;389:1238-52.

7 Saran R, Robinson B, Abbott KC, et al. US renal data system 2018 annual data report: epidemiology of kidney disease in the United States. Am J Kidney Dis 2019;73:A7-8.

8 Yamagata K, Makino H, Iseki K, et al. Effect of behavior modification on outcome in early- to Moderate-Stage chronic kidney disease: a cluster-randomized trial. PLoS One 2016;11:e0151422.

9 Stack AG. Impact of timing of nephrology referral and pre-ESRD care on mortality risk among new ESRD patients in the United States. Am $J$ Kidney Dis 2003;41:310-8.

10 Lin M-Y, Cheng L-J, Chiu Y-W, et al. Effect of national pre-ESRD care program on expenditures and mortality in incident dialysis patients: a population-based study. PLoS One 2018;13:e0198387.

$11 \mathrm{Ku} \mathrm{E}$, Lee BJ, Wei J, et al. Hypertension in CKD: core curriculum 2019. Am J Kidney Dis 2019;74:120-31.

12 Kusaba T, Kimura K. [Management of hypertension in CKD patients]. Nihon Rinsho 2008;66:1747-52.

13 Dare AJ, Fu SH, Patra J, et al. Renal failure deaths and their risk factors in India 2001-13: nationally representative estimates from the million death study. Lancet Glob Health 2017;5:e89-95.

14 Wen CP, Cheng TYD, Tsai MK, et al. All-cause mortality attributable to chronic kidney disease: a prospective cohort study based on 462 293 adults in Taiwan. The Lancet 2008;371:2173-82.

15 McCullough PA, Steigerwalt S, Tolia K, et al. Cardiovascular disease in chronic kidney disease: data from the kidney early evaluation program (KEEP). Curr Diab Rep 2011;11:47-55.

16 Winocour PH. Diabetes and chronic kidney disease: an increasingly common multi-morbid disease in need of a paradigm shift in care. Diabet Med 2018;35:300-5.
17 Peters E, van Elsas A, Heemskerk S, et al. Alkaline phosphatase as a treatment of sepsis-associated acute kidney injury. J Pharmacol Exp Ther 2013;344:2-7.

18 Roughley MJ, Belcher J, Mallen CD. Gout and risk of chronic kidney disease and nephrolithiasis: meta-analysis of observational studies. Arthritis Res Ther 2015;17:90.

19 Zhao G, Huang L, Song M, et al. Baseline serum uric acid level as a predictor of cardiovascular disease related mortality and all-cause mortality: a meta-analysis of prospective studies. Atherosclerosis 2013;231:61-8.

20 Ishihara M, Urushido M, Hamada K, et al. Sestrin-2 and BNIP3 regulate autophagy and mitophagy in renal tubular cells in acute kidney injury. Am J Physiol Renal Physiol 2013;305:F495-509.

21 Borghi C, Rosei EA, Bardin T, et al. Serum uric acid and the risk of cardiovascular and renal disease. J Hypertens 2015;33:1729-41.

22 Bermúdez-López M, Arroyo D, Betriu Àngels, et al. New perspectives on CKD-induced dyslipidemia. Expert Opin Ther Targets 2017;21:967-76.

23 Galvao TF, Araujo MEA, Penha AP, et al. Statins for early stage chronic kidney disease: an overview of reviews. Cardiovasc Hematol Disord Drug Targets 2014;14:205-11.

24 Tangri N, Stevens LA, Griffith J, et al. A predictive model for progression of chronic kidney disease to kidney failure. JAMA 2011;305:1553-9.

25 Hasegawa T, Sakamaki K, Koiwa F, et al. Clinical prediction models for progression of chronic kidney disease to end-stage kidney failure under pre-dialysis nephrology care: results from the chronic kidney disease Japan cohort study. Clin Exp Nephrol 2019;23:189-98.

26 Tonelli M, Muntner P, Lloyd A, et al. Risk of coronary events in people with chronic kidney disease compared with those with diabetes: a population-level cohort study. Lancet 2012;380:807-14.

27 Kornelius E, Chiou J-Y, Yang Y-S, et al. The diabetes shared care program and risks of cardiovascular events in type 2 diabetes. Am J Med 2015;128:977-85. 